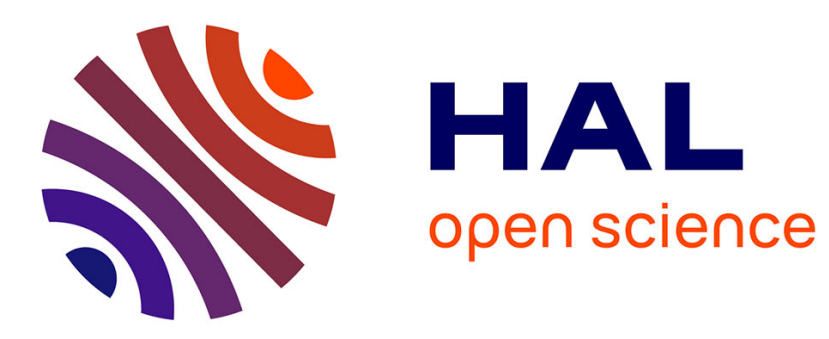

\title{
Wind instruments as time delay systems. Part II: control and estimation
}

Brigitte d'Andréa-Novel, Jean-Michel Coron, Benoît Fabre, Thomas Hélie

\section{To cite this version:}

Brigitte d'Andréa-Novel, Jean-Michel Coron, Benoît Fabre, Thomas Hélie. Wind instruments as time delay systems. Part II: control and estimation. IFAC Workshop on Time Delay Systems, 2009, Sinaia, Romania. pp.7-12. hal-01161416

\section{HAL Id: hal-01161416 https://hal.science/hal-01161416}

Submitted on 8 Jun 2015

HAL is a multi-disciplinary open access archive for the deposit and dissemination of scientific research documents, whether they are published or not. The documents may come from teaching and research institutions in France or abroad, or from public or private research centers.
L'archive ouverte pluridisciplinaire HAL, est destinée au dépôt et à la diffusion de documents scientifiques de niveau recherche, publiés ou non, émanant des établissements d'enseignement et de recherche français ou étrangers, des laboratoires publics ou privés. 


\title{
Wind instruments as time delay systems. Part II: control and estimation *
}

\author{
B. d'Andréa-Novel ${ }^{*}$ J.-M. Coron ${ }^{* *}$ B. Fabre ${ }^{* * *}$ T. Hélie ${ }^{* * * *}$ \\ * Mines-ParisTech, CAOR, 60 bvd Saint-Michel, 75272 Paris Cedex \\ 06, FRANCE (e-mail: brigitte.dandrea-novel@mines-paristech.fr) \\ ** UPMC and IUF, B.C. 187, 4 place Jussieu, 75252 Paris Cedex 05, \\ FRANCE (e-mail: coron@ann.jussieu.fr) \\ *** UPMC, Lutherie, Acoustique, Musique, IJLRA, 11 rue de Lourmel, \\ 75015 Paris, FRANCE (e-mail: benoit.fabre@upmc.fr) \\ **** IRCAM-CNRS UMR 9912, 1 place Igor Stravinsky, 75004 Paris, \\ FRANCE (e-mail: thomas.helie@ircam.fr)
}

\begin{abstract}
In this paper, a modal analysis is performed for the slide flute modeled in [d'Andréa-N et al.(2009)] using the linearized boundary conditions which can also be used to compute the suitable blowing pressure (linked to the mouth delay) and the suitable pipe length (linked to the pipe delay) to obtain a desired fundamental frequency or equivalently a desired note. This will constitute the basis of our control algorithm. Concerning the simplified trumpet-like instrument also described in [d'Andréa-N et al.(2009)] as a so-called nonlinear neutral state space system, we elaborate a local asymptotic observer of the state, the stability of which being proved using Lyapunov techniques.
\end{abstract}

Keywords: Wind instruments; Hyperbolic PDEs; Boundary conditions; Asymptotic observers; Neutral delay system; Lyapunov functions.

\section{INTRODUCTION}

Musical instruments such as wind or bowed string instruments depend crucially on non-linear excitation mechanisms (self-oscillations), driven by low frequency energy supplied by a performer, and are directly linked to the problem of control. The development of theoretical models with a high degree of refinement with respect to the asymptotic regime as well as transient behavior is of great musical importance. Moreover, related numerical simulation methods, as well as joint experimental work are fundamental. In [d'Andréa-N et al.(2009)] we have developed numerical models for a slide flute taking into account a realistic behavior of the air jet dynamics and also a model for a trumpet-like instrument.

-) Concerning the case of the slide flute, we are interested in the present paper to propose a control algorithm to compute the suitable blowing pressure and pipe length to obtain a desired note. This will be done through a modal analysis using the linearized boundary conditions.

-) Concerning the example of the brass instrument, it must be pointed out that the problem of recovering the musician's control parameters from a target sound is sometimes important. Therefore, the inversion of the model appears as a natural tool to cope with this problem which has yet been investigated in the past

\footnotetext{
* Supported by an ANR french project "CONSONNES"
}

[Wold(1987),Hélie et al.(1999)] using control engineering techniques but significant improvements are still needed.

A problem to cope with consists in recovering both the vibro-acoustic state and the musician's control parameters from a unique observation, namely, the sound. The difficulty is increased by the fact that self-sustained instruments are able to generate a large variety of regimes and, possibly, complex regimes such as chaotic ones [Vergez, Rodet(1998)]. Nevertheless, what can be noticed for these systems is the fact that two separate time-scales can be considered. Mainly, vibrating variables $x$ (such as acoustic pressure, reed or lips motion, etc) oscillate at high frequencies compared to the control variables associated to the player's gestures $G$ (pressure in the mouth, reed or lips stiffness, etc).

Then, assuming some usual quantities are measured on the system, such as the radiated pressure at the end of the instrument, called the "output" $y$, the inversion could be performed in two steps :

(P1) the first one consists in recovering the full oscillating internal state $x$ of the instrument from the knowledge of $y$, considering the control $G$ as locally constant. This step is achieved by building a so-called "state observer" in control systems theory;

(P2) the second one consists in computing the accessible parameters $G$ from this observed state. This step could be achieved using adaptive filtering techniques [Ljung(1999)].

This paper addresses in section 2 the control of the slide flute and in section 3 , the first problem (P1) of elaborating 
a state observer for a simplified brass instrument model, assuming that the output $y$ is the pressure at the end of the pipe. Finally we conclude in section 4.

\section{MODAL ANALYSIS OF THE LINEARIZED BOUNDARY CONDITIONS AND CONTROL ALGORITHM FOR THE SLIDE FLUTE}

Let us recall that a model of the slide flute has been elaborated in [d'Andréa-N et al.(2009)]. We have shown that the slide flute is completely described using the control model (1) :

$$
\left\{\begin{array}{l}
\frac{\partial \tilde{\alpha}}{\partial t}(\sigma, t)+\left(\frac{c-\dot{L} \sigma}{L}\right) \frac{\partial \tilde{\alpha}}{\partial \sigma}(\sigma, t)=0 \\
\frac{\partial \tilde{\beta}}{\partial t}(\sigma, t)-\left(\frac{c+\dot{L} \sigma}{L}\right) \frac{\partial \tilde{\beta}}{\partial \sigma}(\sigma, t)=0
\end{array}\right.
$$

the two boundary conditions at the entrance of the resonator (2) and (3) :

$$
\begin{aligned}
& \tilde{\alpha}(0, t)=\tilde{\beta}(0, t)+ \\
& \frac{2 S_{p}}{\rho_{0} c}\left[p_{f}-\rho_{0} l_{c} \frac{d U_{j}}{d t}-\frac{1}{2} \rho_{0} U_{j}^{2}-\frac{c_{1}}{2}(\dot{\tilde{\alpha}}+\dot{\tilde{\beta}})(0, t)-\Delta p\right] \\
& \left(\rho_{0} l_{c}+c_{3} S_{e}\right) \dot{U}_{j}=p_{f}-\frac{1}{2} \rho_{0} U_{j}^{2}+c_{3} \dot{u}_{0}-c_{2} \ddot{u}_{0}
\end{aligned}
$$

and the one at the end of the resonator (4) :

$$
\tilde{\alpha}(1, t)+\tilde{\beta}(1, t)=2 S_{p} \dot{L},
$$

where $c$ denotes the sound velocity in the fluid, $\rho_{0}$ the fluid density at rest, $S_{p}$ the constant section of the pipe which is supposed to be cylindrical, $L$ the pipe length, $U_{j}$ denotes the jet velocity in the flue channel, $l_{c}$ the length of the channel, $S_{e}$ the cross section of the channel at the flue exit and $p_{f}$ the excitation pressure at the entrance of the channel, generated for example by the mouth of the musician.

The physical constants $c_{1}, c_{2}$ and $c_{3}$, as well as the expression of the pressure jump $\Delta_{p}$ responsible of the sound production, are detailed in [d'Andréa-N et al.(2009), Sec. $3]$.

Let us also recall that the Riemann invariants $\alpha(x, t)$, $\beta(x, t)$ are related to the physical flow rate $u(x, t)$ and the acoustic pressure $p(x, t)$ at time $t$ and point $x$ in the pipe through the following relations :

$$
Z=\left(\begin{array}{c}
\alpha \\
\beta
\end{array}\right)=\left(\begin{array}{c}
u+\frac{S_{p}}{\rho_{0} c} p \\
u-\frac{S_{p}}{\rho_{0} c} p
\end{array}\right)
$$

and

$$
\left(\begin{array}{l}
u \\
p
\end{array}\right)=\left(\begin{array}{c}
\frac{\alpha+\beta}{2} \\
\frac{\rho_{0} c(\alpha-\beta)}{2 S_{p}}
\end{array}\right) .
$$

We have denoted $u_{0}=u(0, t)$. Finally, to transform the system in a one with a fixed spatial domain for $\sigma$, i.e. $\sigma \in[0,1]$, we have considered :

$$
x=L \sigma
$$

so that $\tilde{\alpha}(\sigma, t)$ and $\tilde{\beta}(\sigma, t)$ are given by :

$$
\left\{\begin{array}{l}
\tilde{\alpha}(\sigma, t)=\alpha(x, t)=\alpha(L(t) \sigma, t) \\
\tilde{\beta}(\sigma, t)=\beta(x, t)=\beta(L(t) \sigma, t) .
\end{array}\right.
$$

First we perform a modal analysis and then we will present our control algorithm for the flute.

\subsection{Modal analysis}

To compute the natural modes of the system, we have to keep only linear terms in the boundary conditions. Writing $U_{j}, \tilde{\alpha}$ and $\tilde{\beta}$ on the following form, where $U_{0}$ is the steady state value of the jet velocity i.e. :

$$
\begin{gathered}
U_{0}=\sqrt{2 p_{f} / \rho_{0}}, \\
\left\{\begin{array}{l}
U_{j}=U_{0}+U e^{i \omega t} \\
\tilde{\alpha}(x, t)=a e^{i \omega t} e^{-i \omega x / c} \\
\tilde{\beta}(x, t)=b e^{i \omega t} e^{i \omega x / c}
\end{array}\right.
\end{gathered}
$$

replacing $U_{j}, \tilde{\alpha}$ and $\tilde{\beta}$ in (2) and (3) at $x=0$ and (4) at $x=L$ and keeping only linear terms, we have to solve an homogeneous linear system of 3 equations with 3 complex unknowns $(U, a, b)$ of the form :

$$
\begin{aligned}
& A(\omega)\left(\begin{array}{c}
a \\
b \\
U
\end{array}\right)=0, \text { with } A(\omega)= \\
& \left(\begin{array}{lll}
Z_{c}+\kappa\left(\omega, \tau_{l}\right) & -Z_{c}+\kappa\left(\omega, \tau_{l}\right) & 2 \rho_{0}\left(U_{0}+i \omega l_{c}\right) \\
c_{3} i \omega+c_{2} \omega^{2} & c_{3} i \omega+c_{2} \omega^{2} & -2\left(\rho_{0} U_{0}+i \omega c_{0}\right) \\
e^{-i \omega \tau_{p}} & e^{i \omega \tau_{p}} & 0
\end{array}\right)
\end{aligned}
$$

where $\kappa\left(\omega, \tau_{l}\right)=\left(c_{1}+\bar{K} e^{-i \omega \tau_{l}}\right) i \omega, \tau_{p}=\frac{L}{c}$ denoting the delay due to the pipe and $\tau_{l}$ the delay due to the labium considered as a constant ( $W$ being the distance between the flue exit and the labium) :

$$
\tau_{l}=\frac{W}{0.3 U_{0}}
$$

and the constant $\bar{K}$ is obtained by linearizing $\Delta p_{j d}$, the pressure jump due to the jet-drive (see for details [d'Andréa-N et al.(2009), Sec. 3]). In fact, after some computations, we obtain the linearized expression $\Delta \bar{p}_{j d}$ :

$$
\bar{\Delta}_{j d}=\bar{K} \dot{u}_{0}\left(t-\tau_{l}\right) \text {, }
$$

$\bar{K}$ being a constant. See [d'Andréa-N et al.(2008)] or [d'Andréa-N et al.(2009)] for more details.

Therefore, to obtain a non trivial solution, the complex modes $\omega=\lambda+i \epsilon$ must satisfy:

$$
\operatorname{det}(A(\omega))=0 \text {, }
$$

namely, the real numbers $\lambda$ and $\epsilon$ must be solutions of the two real equations:

$$
\left\{\begin{array}{l}
\operatorname{Re}(\operatorname{det}(A(\lambda, \epsilon)))=0 \\
\operatorname{Im}(\operatorname{det}(A(\lambda, \epsilon)))=0 .
\end{array}\right.
$$


In Fig. 1, we can see the $2 \mathrm{D}$-surfaces $\operatorname{Re}(\operatorname{det}(A(\lambda, \epsilon)))=0$ and $\operatorname{Im}(\operatorname{det}(A(\lambda, \epsilon)))=0$ parameterized by $\lambda$ and $\epsilon$.
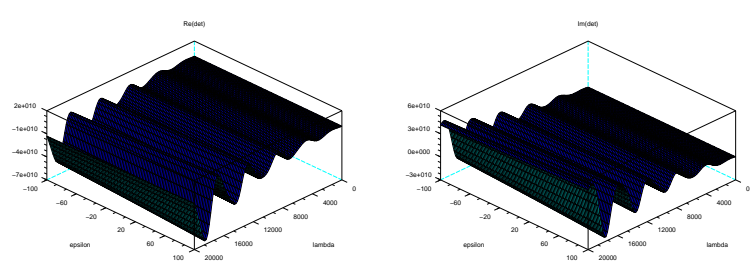

Fig. 1. $\operatorname{Re}(\operatorname{det}(\mathrm{A}))$ and $\operatorname{Im}(\operatorname{det}(\mathrm{A}))$ w.r.t. $\lambda$ and $\epsilon$

For $L=0.20 \mathrm{~m}$, the four first frequencies $f_{i_{c}}, i=1, \cdots, 4$, corresponding to the four first modes $\lambda_{i_{c}}=2 \pi f_{i_{c}}, i=$ $1, \cdots, 4$ are approximately, in $\mathrm{Hz}$ :

$$
f_{1_{c}} \simeq 378, f_{2_{c}} \simeq 1088, f_{3_{c}} \simeq 1922, f_{4_{c}} \simeq 2753 .
$$

The corresponding values of $\epsilon_{i_{c}}$ are, in $s^{-1}$ :

$$
\epsilon_{1_{c}} \simeq-52, \epsilon_{2_{c}} \simeq 165, \epsilon_{3_{c}} \simeq-72, \epsilon_{4_{c}} \simeq-100 .
$$

It can be noticed that:

$$
\frac{f_{2_{c}}}{f_{1_{c}}} \simeq 2.9, \frac{f_{3_{c}}}{f_{1_{c}}} \simeq 5.1, \frac{f_{4_{c}}}{f_{1_{c}}} \simeq 7.3
$$

i.e. the modes are odd multiples of the fundamental frequency, as expected for a closed-open pipe. For negative values of $\epsilon$, the modes are oscillating corresponding to a time growth. Since they are solutions of the linearized problem, the modes calculated here can predict the oscillating frequency during transient state, at low amplitude. After this initial period, the saturation mechanisms may be responsible for a bifurcation in the system.

\subsection{Automatic control algorithm of the flute}

To realize an automatic control law, we have to compute $p_{f}$ (or $U_{0}$ from $(9)$ ) and $L$, or equivalently, the pair of the two delays $t_{a}$ due to the labium and $\tau_{p}$ the delay due to the pipe : $\left(\tau_{l}=\frac{W}{0.3 U_{0}} ; \tau_{p}=\frac{L}{c}\right)$, such that a desired reference mode $\omega_{r}=\lambda_{r}+i \epsilon_{r}$ (or a desired reference frequency $\left.f_{r}=\frac{\lambda_{r}}{2 \pi}\right)$ is obtained. Therefore, the control algorithm can be summarized as follows:

- $\lambda_{r}$ and $\epsilon_{r}$ being chosen, solve the two equations (15) with respect to the unknown variables $\tau_{l_{r}}$ and $\tau_{p_{r}}$, using for example a Newton algorithm.

- The resulting asymptotic jet velocity $U_{0 r}$ will be reached through the servo-valve, asking for a desired steady-state pressure $p_{f_{r}}=\frac{1}{2} \rho_{0} U_{0}^{2}$.

- The resulting length of the pipe $L_{r}$ will be reached, applying the following simple linear control law on the piston:

$$
\dot{L}=-k\left(L-L_{r}\right), k>0 \text {. }
$$

For example, to have $\omega_{r}=2700$, corresponding to $f_{r}=$ $f_{1_{\text {ideal }}}=c / 4 L=430 \mathrm{~Hz}$, solving equations (15) leads to: $\left(U_{0 r}=5.74 \mathrm{~m} / \mathrm{s} ; L_{r}=0.167\right)\left(L_{r}\right.$ has to be compared with $L=0.20)$ or equivalently to $\left(\tau_{l r}=2.3210^{-3} s ; \tau_{p_{r}}=\right.$ $\left.4.8610^{-4} s\right)$.

\subsection{Numerical scheme and simulation results}

The numerical model which will be used for simulation and control is obtained by (1) together with the boundary conditions (2), (4) and (3). It is numerically solved using a first-order un-centered upper scheme for $\tilde{\alpha}$ and down scheme for $\tilde{\beta}$. To be more realistic and take into account visco-thermal losses in the tube, we have also added a small friction term of the form $\mu u$ in Euler equation, $\mu$ being a small friction coefficient. More accurate models exist for visco-thermal damping using non integer time derivative of the flow rate (see e.g. [Matignon(1994)]). In Fig. 2, we can see simulation results obtained with $L=0.20 \mathrm{~m}$ and $p_{f}=5 \mathrm{~Pa}$ during $0.4 \mathrm{~s}$. It can be checked that the jet velocity oscillates with the same frequency as that of the other signals, around a steady value $U_{0}=\sqrt{2 p_{f} / \rho_{0}} \simeq$ $2.89 \mathrm{~m} / \mathrm{s}$.
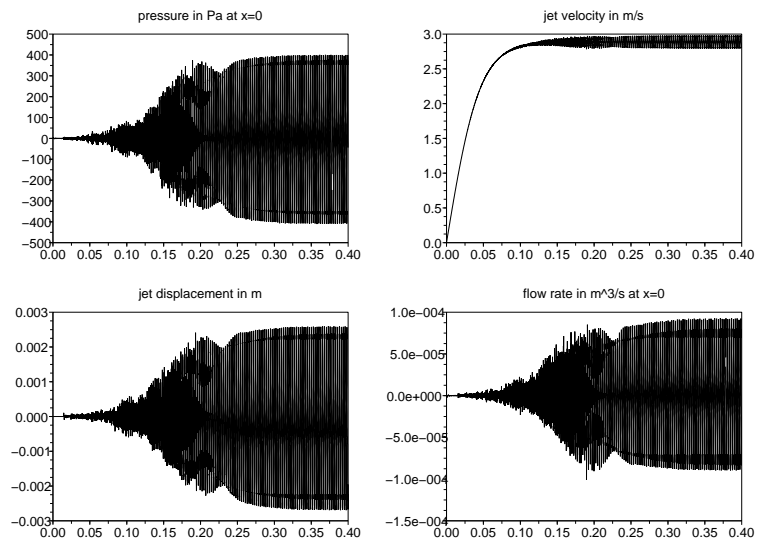

Fig. 2. Simulation results with $p_{f}=5 P a$ and $L=0.20 \mathrm{~m}$

A spectral analysis of the pressure at $x=0$ gives :

$$
f_{1_{s}} \simeq 397 \mathrm{~Hz}, f_{2_{s}} \simeq 1190 \mathrm{~Hz}, f_{3_{s}} \simeq 1982 \mathrm{~Hz}, f_{4_{s}} \simeq 2774 \mathrm{~Hz}
$$

which are very close to the computed values $f_{i_{c}}$ given in (16). The slight difference is probably due to the fact that the computed frequencies $f_{i_{c}}$ are obtained through linearized boundary conditions. Nevertheless, equations (15) constitute a good approximation to compute $L_{r}$ as explained before.

We are aware that the blowing pressure range is lower than in actual pipes. This is mainly due to the simplifications of the jet and source models (see [d'Andréa-N et al.(2009), Sec. 3] which overestimate the jet amplification and sound production at low jet velocities. To solve this problem, we have implemented in [d'Andréa-N, Fabre, Coron(2009)] a numerical filter to obtain a more realistic dynamics of the jet position in the mouth.

Finally, Fig. 3 displays the results we obtain when the player wants to produce scales by sliding the piston. It can be seen that the transient time response is about $0.1 \mathrm{~s}$ which is coherent with the attack transient time observed when the slide is fixed. 

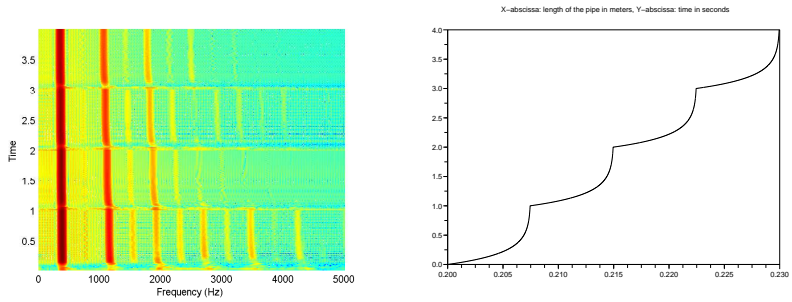

Fig. 3. A down scale with $p_{f}=5 P a$ and $L$ in abscissa from $0.20 \mathrm{~m}$ to $0.23 \mathrm{~m}$

\section{ASYMPTOTIC OBSERVER FOR A BRASS INSTRUMENT}

Let us recall that the model for the trumpet-like instrument is the following neutral system :

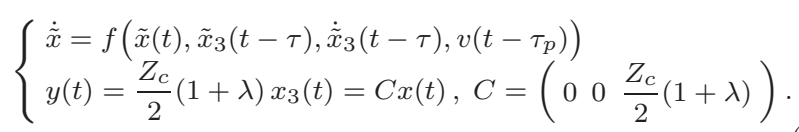

where the state $x$, the input $v$, and the output $y$ are defined as follows : $x_{1}$ represents the lip displacement relative to the equilibrium position $\xi-\xi_{e}, x_{2}$ the lip velocity $\dot{\xi}$, $x_{3}=\alpha_{0}(t)$ the forward propagating wave at the pipe entrance. The input vector $v$ is made of the mouth pressure $p_{m}$ and its time derivative delayed by time $\tau_{p}=\frac{L}{c}$ and $\tau=2 \tau_{p}$ (see [d'Andréa-N et al.(2009)] for details).

As mentioned in the introduction, the problem which is considered here is the construction of an asymptotic state observer, assuming that the physical constant parameters and the mouth pressure $p_{m}$ are known. The idea is to use an extended Kalman filter [Kalman(1960)] type observer elaborated from the nonlinear neutral system presented in [d'Andréa-N et al.(2009)]. This observer will depend on the output error but also on its delayed value.

The gain matrices of the observer will be chosen to stabilize the linear time-varying neutral system governing the linearized equation of the estimation error vector. The proof of stability relies on a suitable Lyapunov function.

\subsection{Definition of the observer}

The following state observer for system (21) is proposed :

$$
\begin{aligned}
\dot{\hat{x}}(t)= & f\left(\hat{x}(t), \hat{x}_{3}(t-\tau), \dot{\hat{x}}_{3}(t-\tau), v\left(t-\tau_{p}\right)\right) \\
& -\Lambda_{1}(y(t)-\hat{y}(t))-\Lambda_{2}(y(t-\tau)-\hat{y}(t-\tau)),
\end{aligned}
$$

where $\Lambda_{1}$ and $\Lambda_{2}$ are $3 \times 1$ gain matrices.

\subsection{Linearized error equation}

Let $e$ denote the estimation error vector:

$$
e=x-\hat{x} \text {. }
$$

The matrices $\Lambda_{1}$ and $\Lambda_{2}$ will be chosen such that the following linearized dynamical equation of the estimation error is locally asymptotically stable :

$$
\dot{e}(t)=\mathbb{A}(t) e(t)+\mathbb{B}(t) e_{3}(t-\tau)+\mathbb{H}(t) \dot{e}_{3}(t-\tau),
$$

$$
\begin{aligned}
\mathbb{A}(X, Y, Z, V) & =\frac{\partial f}{\partial X}(X, Y, Z, V)+\Lambda_{1} C, \\
\mathbb{B}(X, Y, Z, V) & =\frac{\partial f}{\partial Y}(X, Y, Z, V)+\Lambda_{2} C_{3}, \\
\mathbb{H}(X, Y, Z, V) & =\frac{\partial f}{\partial Z}(X, Y, Z, V) .
\end{aligned}
$$

and $\mathbb{A}(t)=\mathbb{A}\left(\hat{x}(t), \hat{x}_{3}(t-\tau), \dot{\hat{x}}_{3}(t-\tau), v\left(t-\tau_{p}\right)\right)$ and similarly for $\mathbb{B}(t)$ and $\mathbb{H}(t)$.

This property will be detailed in theorem 1, the proof of which relying on the following technical lemmas. The proofs of the lemmas are omitted in the present paper but can be found in [d'Andréa-N, Coron et al.(2009)].

Lemma 1. The gain matrix $\Lambda_{2}$ can be chosen such that $\mathbb{B}(t)$ in equation (24) is zero.

Remark 1. From lemma 1, the gain matrix $\Lambda_{2}$, in the observer equation (22) will be time dependent and function of $X=\hat{x}(t), Y=\hat{x}_{3}(t-\tau), Z=\dot{\hat{x}}_{3}(t-\tau), V=v\left(t-\tau_{p}\right)$.

Lemma 2. The matrix $H(t)=\mathbb{H}\left(x(t), x_{3}(t-\tau), \dot{x}_{3}(t-\right.$ $\left.\tau), v\left(t-\tau_{p}\right)\right)$ defined from $(27)$ is such that :

$$
|\mathbb{H}(t)| \leq|\lambda|<1
$$

Lemma 3. Let $\chi>0$. The matrix gain $\Lambda_{1}$ can be chosen such that $\mathbb{A}(t)$ in equation (24) takes the following form :

$$
\mathbb{A}=\left(\begin{array}{cc|c}
0 & 1 & 0 \\
-\omega^{2} & -a & 0 \\
\hline \mathbb{M}_{31} & \mathbb{M}_{32} & -\chi
\end{array}\right)
$$

where $\mathbb{M}(t)=\mathbb{M}\left(\hat{x}(t), \hat{x}_{3}(t-\tau), \dot{\hat{x}}_{3}(t-\tau), v\left(t-\tau_{p}\right)\right)$ with $\mathbb{M}=\frac{\partial f}{\partial X}(X, Y, Z, V)$. This choice of $\Lambda_{1}$ ensures that $\bar{e}=\left(\begin{array}{l}e_{1} \\ e_{2}\end{array}\right)$ is decoupled from $e_{3}$ 's dynamics and satisfies $\dot{\bar{e}}=F \bar{e}$ where the constant matrix $F$ is Hurwitz.

\subsection{Main stability result}

The main stability result is then stated by the following theorem.

Theorem 1. There exist positive constants $\kappa>0$ and $\eta>0$, such that for every solution of equation (24), the following inequality holds :

$$
\begin{aligned}
& \left(e_{1}^{2}(t)+e_{2}^{2}(t)+e_{3}^{2}(t)+\int_{t-\tau}^{t}{\dot{e_{3}}}^{2}(s) d s\right) \leq \\
& \kappa e^{-\eta t}\left(e_{1}^{2}(0)+e_{2}^{2}(0)+e_{3}^{2}(0)+\int_{-\tau}^{0} \dot{e_{3}^{2}}(s) d s\right) .
\end{aligned}
$$

Proof : From lemma 3, $F$ being Hurwitz, define the symmetric positive definite matrix $P$ solution of the matrix Lyapunov equation [d'Andréa-N, Cohen de Lara(1994)] :

$$
F^{T} P+P F=-I_{2 \times 2}
$$

$I_{2 \times 2}$ denoting the $2 \times 2$ identity matrix.

Now, consider the following Lyapunov function candidate where $C>0, K>0$ and $\nu>0$ are suitable positive constants : 


$$
V=C \frac{e_{3}^{2}}{2}+K \bar{e}^{T} P \bar{e}+\int_{t-\tau}^{t}{\dot{e_{3}}}^{2}(s) e^{-\nu(t-s)} d s .
$$

If the constant $C$ is chosen to be equal to $C=2 \chi$, where $\chi>0$ is defined in lemma 3 , the time derivative of $V$ along the solutions of the dynamical equations of the linearized observation error (24) can be written as follows :

$$
\dot{V}=T_{1}+T_{2}+T_{3}
$$

where

$$
\begin{aligned}
T_{1}= & -\chi^{2} e_{3}^{2}-{\dot{e_{3}}}^{2}(t-\tau)\left(e^{-\nu \tau}-H_{3}^{2}(t)\right) \\
& -K\left(e_{1}^{2}+{\dot{e_{2}^{2}}}^{2}\right) \\
& -\nu \int_{t-\tau}^{t}{\dot{e_{3}}}^{2}(s) e^{-\nu(t-s)} d s, \\
T_{2}= & \left(M_{31} e_{1}+M_{32} e_{2}\right)^{2}, \\
T_{3}= & 2 H_{3}\left(M_{31} e_{1} \dot{e_{3}}(t-\tau)+M_{32} e_{2} \dot{e_{3}}(t-\tau)\right) .
\end{aligned}
$$

Notice that for all $\epsilon>0$ and for all $e_{1}, e_{2}$ and $e_{3}(t-\tau)$, the following inequalities hold for some positive constants $R_{1}$ and $R_{2}$ :

$$
T_{3} \leq \frac{1}{\epsilon}\left(e_{1}^{2}+e_{2}^{2}\right)+R_{1} \epsilon{\dot{e_{3}}}^{2}(t-\tau)
$$

and

$$
T_{2} \leq R_{2}\left(e_{1}^{2}+e_{2}^{2}\right) .
$$

Moreover, from lemma 2 , if the constant $\nu$ in $V$ is chosen sufficiently small, $\left(e^{-\nu \tau}-H_{3}^{2}(t)\right) \geq e^{-\nu \tau}-\lambda^{2} \geq \delta>0$, for all $t$ and $\tau \in[0,2 L / c]$, so that :

$$
\begin{aligned}
\dot{V} \leq & -\chi^{2} e_{3}^{2}-\delta{\dot{e_{3}}}^{2}(t-\tau) \\
& -\left(K-R_{2}\right)\left(e_{1}^{2}+e_{2}^{2}\right) \\
& +\frac{1}{\epsilon}\left(e_{1}^{2}+e_{2}^{2}\right)+R_{1} \epsilon \dot{\dot{e}_{3}}{ }^{2}(t-\tau) \\
& -\nu \int_{t-\tau}^{t}{\dot{e_{3}}}^{2}(s) e^{-\nu(t-s)} d s .
\end{aligned}
$$

Choose

$$
\epsilon=\frac{\delta}{2 R_{1}}
$$

which leads to :

$$
\begin{aligned}
\dot{V} \leq & -\chi^{2} e_{3}^{2}-\frac{\delta}{2} \dot{e_{3}}{ }^{2}(t-\tau) \\
& -\left(K-R_{2}-\frac{2 R_{1}}{\delta}\right)\left(e_{1}^{2}+e_{2}^{2}\right) \\
& -\nu \int_{t-\tau}^{t} \dot{e_{3}}{ }^{2}(s) e^{-\nu(t-s)} d s .
\end{aligned}
$$

Then, for $K=R_{2}+\frac{4 R_{1}}{\delta}$ and for a suitable positive constant $\eta>0$, the following inequality holds:

$$
\dot{V} \leq-\eta V
$$

which ends the proof. $\diamond$

Notice that the weight $e^{ \pm \nu x}$ in the integral term of the Lyapunov function is essential to get a strict Lyapunov function. It is similar to the one introduced by Castelan and Infante [Castelan, Infante(1979)] for matrix difference differential equations and also by Coron [Coron(1999)] to stabilize the Euler equation of incompressible fluids. More recently, it has also been used for linear symmetric hyperbolic systems [Xu, Sallet(2002)] and for exponential stabilization of one-dimensional nonlinear hyperbolic systems [Coron et al.(2007), Coron et al.(2008)].

\subsection{Simulation results}

Observation results have been performed on outputs $y$, simulated using an Euler explicit scheme on (21). To be more realistic, measurement gaussian noises have been added to $y$.

Since the observer (22) is local, the initial conditions of $\hat{x}$ must be chosen sufficiently close to the actual initial condition $x(0)=0$.

To test the robustness of this observer, several deviations on initial conditions and several noise measurements have been considered.

It is important to notice that, due to lemma 3 , the characteristic damping of $\bar{e}=\left[e_{1}, e_{2}\right]^{T}$ is $a$. Moreover, the time constant of the observer is governed by $a$ and $\chi$. The arbitrary damping coefficient $\chi$ in $\mathbb{A}$ can be preferentially chosen greater than the fixed constant $a$. In practice, it is natural to choose $\chi \approx a$ (or slightly greater than $a$ ).

In a quasi ideal situation with initial conditions close to the actual ones and low measurement noise, the proposed observer $\hat{x}$ has a pretty good behavior and converges rapidly towards $x$ and the measurement noises are weakened quite well. For initial conditions quite far from the ideal ones (e.g. $\frac{Z_{c}}{2}(1+\lambda) e_{3}(0)$ is about $20 \%$ of the mouth pressure $\left.p_{m}\right)$, the obtained results show that the transient behavior is longer but the observer succeeds in retrieving the real value of the state (see [d'Andréa-N, Coron et al.(2009)]). Finally, Fig. 4 illustrates the robustness of the local observer with respect to larger measurement noises $\left(\sigma_{\text {noise }} / p_{m}\right.$ is about $\left.3 \%\right)$. In this case, the limits of the observer are reached.

Notice that since $y=\frac{Z_{c}}{2}(1+\lambda) x_{3}$ is measured, in a simpler approach, it is not necessary to estimate $x_{3}$ and it could have been sufficient to elaborate observer on $x_{1}, x_{2}$ only. Nevertheless, since there are noise measurements, the total observer proposed here proves to be more robust. Indeed in Fig. 4, the noise on $\widehat{x}_{3}$ has been significantly reduced compared to that on $y$.

\section{CONCLUSION}

We have shown in this paper the interest of using tools from the theory of time delay systems to deal with some control and estimation problems for wind instruments. Two illustrative examples have been considered. First, we have addressed the problem of controlling a slide flute and elaborated a control algorithm through a modal analysis, to compute the blowing pressure and the piston velocity. On the experimental prototype which is under development, a real time spectral analysis will be performed so that we will be able to adjust the length $L$ of the tube around the estimated value $L_{r}$ to improve the accuracy of the resulting frequency.

Concerning the case of the brass instrument, it has been proved using Lyapunov function techniques on neutral 

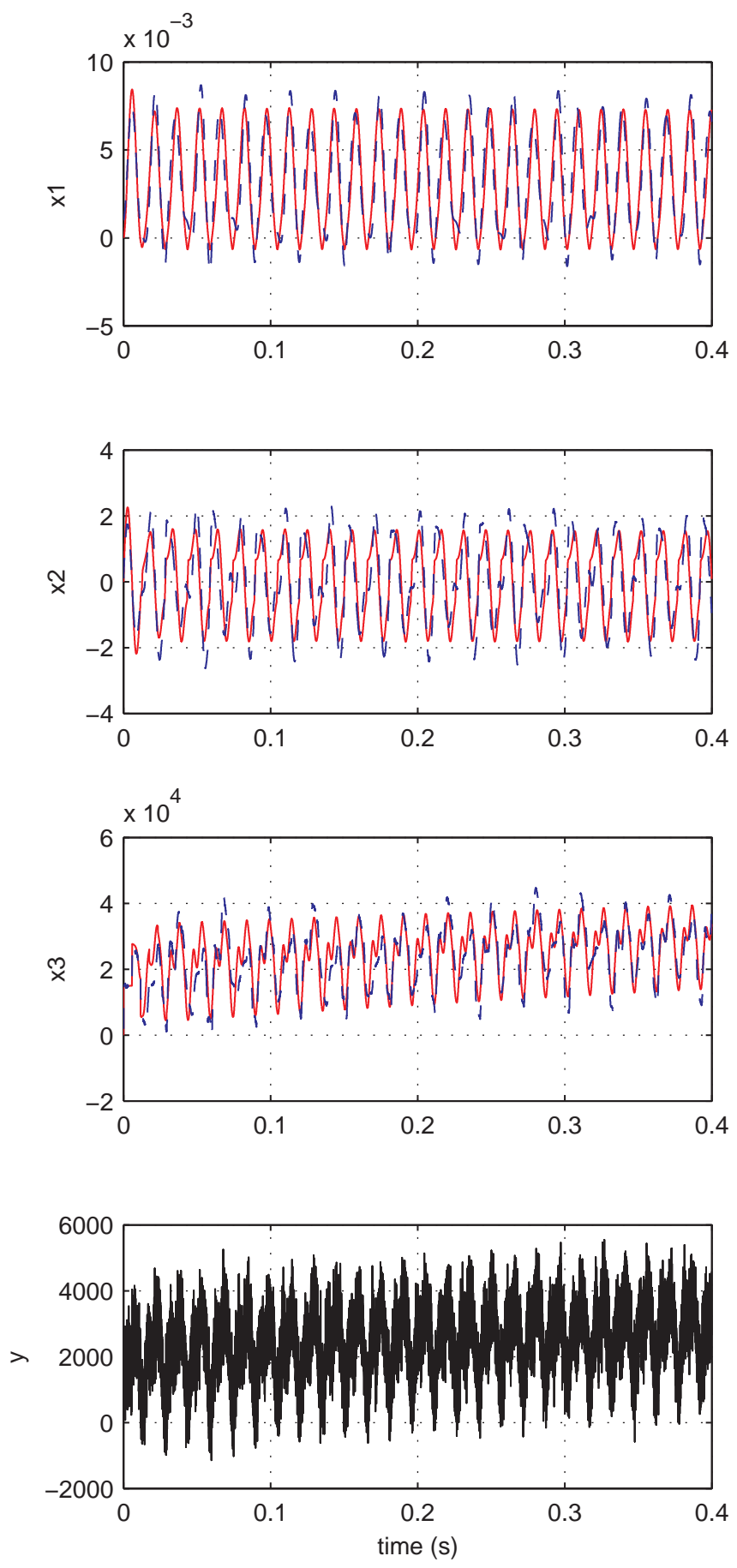

Fig. 4. Simulation of the system (-) and the observer (--) from the measured output $y(-)$. The simulations are performed for the following parameters: $a=150 \mathrm{~s}^{-1}$, $\chi=160 \mathrm{~s}^{-1}, L=1, p_{m}=1.5 e 4 P a, \omega=535 s^{-1}$, quasi-ideal initial conditions $\hat{x}(0)=[1 e-3,1 e-$ $\left.1, \frac{y(0)}{1+\lambda}\right]^{T}$ and large noise $\sigma_{\text {noise }} / p_{m}=4 e-2$.

systems that the observer gains can be tuned to ensure the asymptotic stability of the linearized estimation error equation. Moreover, although the proof ensures only a local stabilization, simulation results exhibit good robustness properties with respect to wrong initial conditions and significant noises on the measured output.
A future work will concern the second problem (P2) described in the introduction, that is, computing the "player's gestures" from the measured output and the proposed observer. It could be processed by using adaptive filtering techniques, and thus, would complete the global inversion problem.

\section{ACKNOWLEDGEMENTS}

The authors would like to thank Silviu Niculescu for his helpful advices concerning time delay systems.

\section{REFERENCES}

[d'Andréa-N, Cohen de Lara(1994)] B. d'Andréa-Novel, M. Cohen de Lara, Commande linéaire des systèmes dynamiques. Masson, Paris, 1994.

[d'Andréa-N et al.(2008)] B. d'Andréa-Novel, B. Fabre, J.-M. Coron, Modeling and control of a slide flute. Proc. of 16th Mediterranean Conference on Control and Automation, pages 15681573, Ajaccio, France, June 2008.

[d'Andréa-N et al.(2009)] B. d'Andréa-Novel, J.-M. Coron, B. Fabre, T. Hélie, Wind instruments as time delay systems. Part I: modeling. Proc. of TDS Conference, Sinaia, Romania, September 2009.

[d'Andréa-N, Fabre, Coron(2009)] B. d'Andréa-Novel, B. Fabre, J.M. Coron, Modeling and automatic control of a slide-flute. ACTA Acustica, submitted, July 2009.

[d'Andréa-N, Coron et al.(2009)] B. d'Andréa-Novel, J.-M. Coron, B. Fabre, T. Hélie, Asymptotic observers for a simplified brass instrument model. ACTA Acustica, submitted, July 2009.

[Castelan, Infante(1979)] W.-B. Castelan, E.-F. Infante, A Liapunov functional for a matrix neutral difference-differential equation with one delay. Journal of Mathematical Analysis and Applications, Volume 71, Issue 1, pages 105-130, September 1979.

[Coron(1999)] J.-M. Coron, On the null asymptotic stabilization of the two-dimensional incompressible Euler equations in a simply connected domain. SIAM Journal of Control and Optimization, 37(6), pages 1874-1896, 1999.

[Coron et al.(2007)] J.-M. Coron, B. d'Andra-Novel, G. Bastin, A strict Lyapunov function for boundary control of hyperbolic systems of conservation laws. IEEE Transactions on Automatic Control, 52(1), pages 2-11, January 2007.

[Coron et al.(2008)] J.-M. Coron, G. Bastin, B. d'Andra-Novel, Dissipative boundary conditions for one dimensional nonlinear hyperbolic systems. SIAM Journal of Control and Optimization, 47(3), pages 1460-1498, 2008.

[Hélie et al.(1999)] T. Hlie, C. Vergez, J. Lvine, X. Rodet, Inversion of a physical model of a trumpet. proc. of the Conference on Decision and Control, Phoenix, Arizona, Vol. 38.3, pages 25932598, 1999.

[Kalman(1960)] R. Kalman, A new approach to linear filtering and prediction problems. J. of Basic Engineering, pages 35-45, 1960.

[Ljung(1999)] L. Ljung, System Identification-Theory For The User. 2nd edition, Prentice-Hall, Upper Saddle River, N.J., 1999.

[Matignon(1994)] D. Matignon, Représentation en variables d'état de modèles de guide d'ondes avec dérivation fractionnaire. $P h D$ Thesis, Université de Paris-Sud, Orsay, France, 1994.

[Vergez, Rodet(1998)] C. Vergez, X. Rodet, Bifurcation sequence in a physical model of trumpet-like instruments: from a fixed point to chaos. proc. NOLTA, IEICE, Crans Montana, Switzerland, Vol. 2, pages 751-754, 1998.

[Wold(1987)] E.H. Wold, Nonlinear parameter estimation of acoustic models. PhD thesis, Computer Science Division, Berkeley University of California, 1987.

[Xu, Sallet(2002)] C.-Z. Xu, G. Sallet, Exponential stability and transfer functions of processes governed by symmetric hyperbolic systems. ESAIM Control Optimisation and Calculus of Variations, 7, pages 421-442, 2002. 